\title{
Utilization of genetic counseling after diagnosis of a birth defect-trends over time and variables associated with utilization
}

\author{
Anne Glynn, BSW, Grad Dip Gen Couns, Veronica Collins, PhD, and Jane Halliday, PhD
}

\begin{abstract}
Purpose: To examine utilization of genetic counseling after diagnosis of a birth defect in 2004, and trends in utilization from 1991 to 2004. Methods: Birth defects data for births in 2004 were linked to genetic counseling data to determine utilization of genetic counseling in Victoria, Australia. Variability in utilization was determined according to the need for genetic counseling (as indicated by the particular birth defect), and demographic and perinatal variables. Trends in utilization were determined by comparing 2004 data with that of earlier studies using the same data sources for birth defects cohorts in 1991, 1993, and 1995. Results: Frequency of overall utilization was $20 \%$ and was not affected by maternal country of birth, socioeconomic advantage/disadvantage, or region of residence. Higher-than-average utilization was strongly predicted by "high-need" (48.4\%), infant death (stillbirth 50\%, postnatal death $50.4 \%$ ), or birth in a tertiary level hospital (28.5\%). There was an upward trend in the proportion of the high-need group using genetic counseling, progressively increasing from $39.7 \%$ in the 1991 cohort to $42.5 \%$ in the 1993 cohort, $46.5 \%$ in the 1995 cohort, to a high of $48.4 \%$ in the 2004 cohort. Conclusions: Utilization by those who most need it has gradually increased from 1991 to 2004, with no inequity of access apparent in the most recent cohort. Further studies are needed to determine whether high-need families not using genetic counseling are not doing so because of chance or choice. Genet Med 2009:11(4): 287-293.
\end{abstract}

Key Words: congenital malformations, birth defects, genetic counseling, utilization

$T^{1}$ he cause of birth defects is often unknown, but when the cause is known it is usually at least partly genetic. ${ }^{1}$ Genetic information, however, is often complex, needing extensive discussion to work through the information and issues relevant to the individual. ${ }^{2}$ Genetic counseling about birth defects can provide such information and discussion. It can help make genetic diagnoses, provide information about the nature of the defect and preventive and therapeutic measures available, provide information about recurrence risk and options for dealing with this risk, and provide support. ${ }^{3}$ It is therefore appropriate that parents of a fetus or infant diagnosed with a birth defect have access to genetic counseling services.

Some previous research looking at utilization of genetic counseling services by parents of infants/fetuses with birth defects has been done by linking birth defects data to genetic

From the Department of Public Health Genetics, Murdoch Childrens Research Institute, The Royal Children's Hospital, Melbourne, Australia.

Anne Glynn, Murdoch Childrens Research Institute, Royal Children's Hospital, Flemington Road, Parkville, Victoria 3052, Australia. E-mail: anne.glynn@mcri.edu.au.

Disclosure: The authors declare no conflict of interest.

Submitted for publication October 5, 2008.

Accepted for publication November 24, 2008.

DOI: $10.1097 /$ GIM.0b013e3181973913 counseling data. ${ }^{4-9}$ This research has shown that genetic counseling services provided in the public health system seem to be underused by parents who have experienced the diagnosis of a birth defect in an infant/fetus, finding that $<20 \%$ of the parents had attended for genetic counseling when all birth defects were included in the analysis. Even when the birth defects were categorized according to the need for genetic counseling/type of birth defect, those in higher need categories only had a utilization rate of 26-63\%. Here, in the State of Victoria, Australia, it has previously been found that only $40-43 \%$ of "high-need" families had used genetic counseling, despite services being provided to families free of charge. ${ }^{6}$

Although some parents may choose not to have genetic counseling simply because it is not of interest or relevance to them, or because their treating clinician has performed this function, the data suggest that various perinatal or demographic factors may influence this under-utilization. The type of birth defect, ,5-9 infant survival status, $,, 5,7-9$ previous affected sibs, 5,7 previous live births, ${ }^{5}$ maternal age, ${ }^{6,9}$ maternal race/ethnicity, ${ }^{9}$ region of residence relative to genetic clinic, ${ }^{4,8}$ and type of birth hospital, ${ }^{6}$ have been found to influence utilization.

In Victoria, a government-funded genetic counseling service has been available since 1988. All parents of a child with a genetic disorder and/or birth defect are eligible to receive genetic counseling through this service at no financial cost to the family. An assessment of the utilization of this state-wide genetic counseling service has been done on two previous occasions by linking the records of the Victorian Birth Defects Register (VBDR) to those of the genetics service (Genetic Health Services Victoria). ${ }^{6,10}$ The genetics service has grown in recent years with the introduction of genetics clinics in at least five additional regional/rural towns and four additional metropolitan hospitals that may have improved the utilization of genetic counseling. Utilization could also be affected by various demographic and perinatal variables. These are examined in this study to facilitate the identification of factors that may impact on peoples' access to genetic counseling, and, if appropriate, point the way for corrective action

\section{MATERIALS AND METHODS}

\section{Primary outcome of interest and record linkage}

The primary outcome of interest was utilization of genetic counseling by families where a birth has been registered on the VBDR. Utilization was measured by linking records from the VBDR with state-wide genetic counseling data. Computerized record linkage methods (Linkage Wiz v4.1) were used to match the two data sources, and all computer matches were viewed to ensure that the genetic counseling consultation was related to the birth defect. The Human Research Ethics Committee of the Department of Human Services Victoria granted ethics approval for this project. 


\section{Sources of data}

The birth defects data were obtained from the VBDR, which is a state-wide, population-based surveillance system held by the Victorian Perinatal Data Collection Unit at the Department of Human Services. Since 1983, the VBDR has collected data from multiple sources about birth defects occurring in live births, stillbirths, and terminations of pregnancy for fetal abnormality. Notifiable birth defects are structural defects or chromosomal abnormalities present at birth, inborn errors of metabolism, hematological disorders, congenital infections, neoplasms, and developmental delay (associated with a syndrome). Notification to the VBDR is voluntary beyond the perinatal period, but notification rates are high, particularly for birth defects due to chromosomal abnormalities and birth defects apparent at birth. ${ }^{11}$ Data for births (including stillbirths) at 20 weeks gestation or more and data for terminations of pregnancy for fetal abnormality (at any gestation) occurring in 2004 were extracted for this study $(n=2939)$. Data for an additional 289 cases with isolated minor malformations of undescended testis, inguinal hernia, or hydrocele were excluded, as referral for genetic counseling would not be appropriate for an isolated occurrence of any of these conditions, which were also excluded in the previous Victorian studies. Data on some variables were not recorded for all cases; therefore, the total cases in subgroups for those variables is $<2939$.

The genetic counseling data were obtained from the central database of Genetic Health Services Victoria that provided services at 13 metropolitan and 10 rural/regional locations throughout the state during the study period. Data from approximately 29,000 genetic consultations occurring between June 2003 and December 2006 inclusive were obtained. The 2003 data were included so that any relevant genetic counseling sessions occurring during the affected pregnancy, because of the prenatal diagnosis of a birth defect or a previously affected sibling/relative, would be counted.

\section{Variables potentially associated with utilization of genetic counseling}

Variables obtained directly from the VBDR data included maternal age, maternal country of birth, infant survival status, and region of residence. In addition, variables relating to "need" for genetic counseling, type of birth hospital, socioeconomic advantage/disadvantage, and location of nearest genetic counseling clinic relative to birth hospital were derived from the birth defects data as outlined below.

\section{Need for genetic counseling}

Each birth defect was categorized as having a "high," "moderate," or "low" need for genetic counseling. The categorization of need was based on that previously developed by a medical officer and medical geneticist ${ }^{6}$ :

\section{High need}

All single gene and chromosomal disorders, neural tube defects, dwarfing conditions, and patterns of malformation associated with known syndromes.

\section{Moderate need}

Polygenic conditions, possible syndromes, and clefting conditions that are not part of a syndrome.

\section{Low need}

Includes all other conditions unlikely to have a strong genetic component and without a known recurrence risk.

\section{Type of birth hospital}

The 78 maternity hospitals at which births or terminations of pregnancy occurred were reclassified as "tertiary," "metropolitan public," "private," or "country." Metropolitan hospitals that cater to both public and private patients were classified as metropolitan public.

\section{Socioeconomic advantage/disadvantage}

We used the socioeconomic index of relative advantage/ disadvantage, produced by the Australian Bureau of Statistics (ABS), ${ }^{12}$ based on postcode of residence. The index was derived by the ABS from 2001 census data using data on education, income, internet access, housing, type of employment, family structure, and unemployment for people residing in each postcode area, to produce an index value for that postcode. Each case in the birth defects data was given the ABS socioeconomic index value applicable for their postcode of residence. ABS population reference values for Victoria were then used to determine the relative advantage/disadvantage associated with that index value, ranging from the relatively most disadvantaged quartile (lowest index values) to the relatively most advantaged quartile (highest index values).

\section{Statistical analysis}

Descriptive statistics included frequencies and cross-tabulations. Data from the 2004 cohort were compared with that from two earlier studies to examine trends in utilization over time and the $\chi^{2}$ test for trend was used to assess statistical evidence for observed trends.

Associations between utilization of genetic counseling and the following variables were assessed: need for genetic counseling, maternal age, infant survival status at hospital discharge, type of birth hospital, area of residence (rural or metropolitan), socioeconomic advantage/disadvantage, and maternal country/ region of birth. The statistical evidence for observed associations was assessed using $\chi^{2}$ tests of association and trends across ordered categories were examined with the $\chi^{2}$ test for trend. Where there were more than two categories in the variable potentially associated with the utilization of genetic counseling, the degrees of freedom have been given with the associated $\chi^{2}$ statistic and $P$ value.

Variables associated with utilization in the bivariate analysis with $P<0.10$ were included in logistic regression models. To examine the independent effect of maternal age, type of birth hospital, and infant survival status on utilization of genetic counseling, logistic regression models were calculated for both the whole cohort and the high-need group. Category of need was also included as an independent variable in the model for the whole cohort.

\section{RESULTS}

Overall, 20\% (588 of 2939) of families who had a birth or termination of pregnancy in 2004 with a registered birth defect were seen for genetic counseling related to that birth defect. Table 1 summarizes utilization according to the need for counseling. Utilization was highest, at $48.4 \%$, for those who are classified as high-need and there was a highly significant trend in proportions across the need categories $\left(\chi^{2}\right.$ trend $=$ 468, $P<0.001)$.

The overall proportion of those using genetic counseling in the 2004 cohort was similar to that found for the 1995 cohort (Table 2) but there was an increasing trend across the four time points $\left(\chi^{2}\right.$ trend $\left.=10.9, P=0.001\right)$. Although the overall 
Table 1 Utilization of genetic counseling categorized by "need" by 2004 birth defects cohort ascertained from Victorian Birth Defects Register

\begin{tabular}{lccc}
\hline Category of need & $\begin{array}{c}\text { Number registered with one or more birth } \\
\text { defects }\end{array}$ & $\begin{array}{c}\text { Number used genetic } \\
\text { counseling }\end{array}$ & \% Used genetic counseling (95\% CI) \\
\hline High & 715 & 346 & $48.4(44.7-52.1)$ \\
Moderate & 1229 & 195 & $15.9(13.9-17.9)$ \\
Low & 995 & 47 & $4.7(3.4-6.0)$ \\
Total & 2,939 & 588 & $20.0(18.6-21.4)$ \\
\hline
\end{tabular}

Table 2 Trends in utilization of genetic counseling by birth defects cohorts 1991-2004 ascertained from Victorian Birth Defects Register

\begin{tabular}{lccc}
\hline $\begin{array}{l}\text { Birth defects } \\
\text { cohort }\end{array}$ & $n$ & $\begin{array}{c}\text { Number used } \\
\text { genetic } \\
\text { counseling }\end{array}$ & $\begin{array}{c}\text { \% Used genetic } \\
\text { counseling } \\
(95 \% \text { CI) }\end{array}$ \\
\hline 1991 & 2254 & 380 & $16.9(15.4-18.4)$ \\
1993 & 2154 & 349 & $16.2(14.6-17.8)$ \\
1995 & 2375 & 472 & $19.9(18.2-21.6)$ \\
2004 & 2939 & 588 & $20.0(18.6-21.4)$ \\
\hline
\end{tabular}

For each category of 'need', utilization is shown as a percentage of total birth defect registrations in that category and year (shown by the numbers above bars).

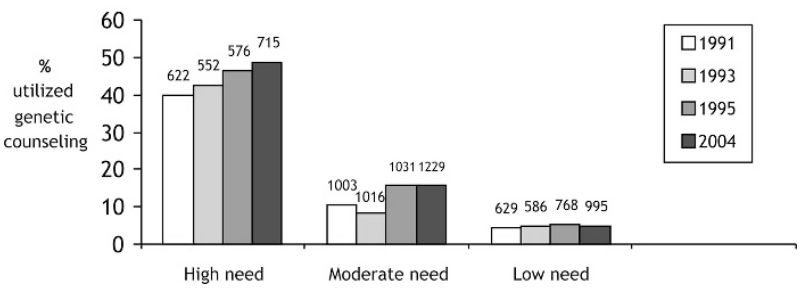

Fig. 1. Trends in utilization of genetic counseling by birth defects cohorts (ascertained from Victorian Birth Defects Register) 1991-2004, according to "need."

proportion of the 2004 cohort using genetic counseling was similar to the 1995 cohort, the actual number of families using genetic counseling has increased markedly, from 472 to 588 .

Figure 1 shows the variation in utilization of genetic counseling over time, for each category of need. There is a continuing upward trend in the proportion of the high-need group using genetic counseling, from $39.7 \%$ in the 1991 cohort to $48.4 \%$ in 2004 cohort $\left(\chi^{2}\right.$ trend $\left.=9.3, P=0.002\right)$. A significant trend in utilization over time was also evident in the moderate-need group $\left(\chi^{2}=20.7, P<0.001\right)$, although utilization in 2004 was similar to that seen in 1995. The proportion of the low-need group using genetic counseling has remained fairly constant over time $\left(\chi^{2}\right.$ trend $=0.01, P=0.92$ ).

Table 3 summarizes utilization of genetic counseling by the 2004 birth defects cohort according to maternal/fetal variables. Maternal age showed a weak association with utilization of genetic counseling in the total cohort with the oldest age-group showing the highest utilization. Evidence for an age association was stronger in the high-need category but in this cohort the $40+$ age group had the lowest utilization. Hospital of birth and infant survival status showed significant associations with utilization of genetic counseling in both the total and the high-need cohorts. The termination of pregnancy ( $<20$ weeks) group had relatively low utilization when compared with the other groups where the infant did not survive. The majority $(83.1 \%)$ of the high-need termination of pregnancy $(<20$ weeks) group who did not use genetic counseling had a diagnosis of Down syndrome or other chromosomal trisomy, or neural tube defect (data not shown). Residents of metropolitan and rural/regional areas had similar levels of utilization of genetic counseling and there was no increasing trend in utilization with socioeconomic advantage (Table 3).

Figure 2 shows utilization of genetic counseling according to maternal country of birth, 2004 birth defects cohort. In the total cohort, mothers from most countries/regions used genetic counseling at a level close to the overall average of $20 \%\left(\chi^{2}{ }_{7 \mathrm{df}}=10\right.$, $P=0.191)$. Similarly for the high-need cohort, utilization was close to the high-need average of $48 \%$ for most maternal countries/regions of birth $\left(\chi_{7 \mathrm{df}}^{2}=7.3, P=0.396\right)$.

Table 4 presents the results of logistic regression models determining the independent association of the variables associated with utilization from bivariate analyses. For the whole cohort, need for counseling was the most important predictor of utilization. After adjusting for need for counseling, giving birth in a tertiary level hospital was strongly associated with increased utilization compared with all other hospital types. The infant's survival status was also strongly associated with utilization such that those experiencing a stillbirth or a postnatal death were more than twice as likely to have genetic counseling compared with other infant survival categories. Evidence for a maternal age effect was fairly weak.

For the high-need cohort, similar results were found, except that the association between having a stillbirth and an increased likelihood of utilization of genetic counseling seen in the whole cohort was not evident. Furthermore, termination of pregnancy $(<20$ weeks $)$ was associated with a much reduced likelihood of having genetic counseling compared with cases where the infant survived.

\section{DISCUSSION}

The linkage and analysis of routinely collected data builds on that from two earlier studies in Victoria to give some insight into trends in the utilization of genetic counseling over time, and into the impact of a range of variables on the level of utilization. The overall proportion of families using genetic counseling was $20 \%$, which was at the high end of the range found in previous studies in Europe, United States, and Australia. ${ }^{5-9}$ An upward trend in utilization in Victoria was evident, particularly compared with the 1991 and 1993 cohorts. The proportion of the high-need group using genetic counseling has steadily increased 
Table 3 Percent utilization of genetic counseling according to maternal/infant variables, 2004 birth defects cohort ascertained from Victorian Birth Defects Register

\begin{tabular}{|c|c|c|c|c|}
\hline & \multicolumn{2}{|c|}{ Whole cohort (all needs) } & \multicolumn{2}{|c|}{ High-need group } \\
\hline & $n$ & $\%$ Used & $n$ & $\%$ Used \\
\hline \multicolumn{5}{|l|}{ Maternal age } \\
\hline$<20$ & 76 & 21.1 & 15 & 60.0 \\
\hline $20-24$ & 319 & 16.9 & 45 & 48.9 \\
\hline $25-29$ & 703 & 16.8 & 107 & 43.0 \\
\hline $30-34$ & 998 & 21.0 & 205 & 59.5 \\
\hline $35-39$ & 632 & 21.8 & 218 & 45.9 \\
\hline \multirow[t]{2}{*}{$\geq 40$} & 208 & 25.0 & 123 & 38.2 \\
\hline & \multicolumn{2}{|c|}{$\chi_{5 \mathrm{df}}^{2}=12 ; P=0.039$} & \multicolumn{2}{|c|}{$\chi_{5 \mathrm{df}}^{2}=18 ; P=0.003$} \\
\hline \multicolumn{5}{|l|}{ Type of birth hospital } \\
\hline Tertiary & 965 & 28.5 & 232 & 69.0 \\
\hline Metro public & 617 & 15.7 & 130 & 46.9 \\
\hline Private & 747 & 15.5 & 198 & 35.4 \\
\hline \multirow[t]{2}{*}{ Country } & 609 & 16.4 & 155 & 35.5 \\
\hline & \multicolumn{2}{|c|}{$\chi^{2}=65 ; P<0.001^{a}$} & \multicolumn{2}{|c|}{$\chi^{2}=58, P<0.001^{a}$} \\
\hline \multicolumn{5}{|l|}{ Infant survival status at hospital discharge } \\
\hline Termination of pregnancy ( $<20 \mathrm{wks})$ & 335 & 36.4 & 291 & 34.7 \\
\hline Stillborn $^{b}$ & 128 & 50.0 & 67 & 53.7 \\
\hline Postnatal death ${ }^{c}$ & 113 & 50.4 & 51 & 68.6 \\
\hline \multirow[t]{2}{*}{ Alive } & 2,363 & 14.6 & 306 & 56.9 \\
\hline & \multicolumn{2}{|c|}{$\chi_{3 \mathrm{df}}^{2}=237 ; P<0.001$} & \multicolumn{2}{|c|}{$\chi_{3 \mathrm{df}}^{2}=40, P<0.001$} \\
\hline \multicolumn{5}{|l|}{ Area of Residence } \\
\hline Metropolitan & 2,151 & 20.0 & 531 & 48.4 \\
\hline \multirow[t]{2}{*}{ Rural/regional } & 713 & 20.2 & 168 & 48.8 \\
\hline & \multicolumn{2}{|c|}{$\chi^{2}=0.01 ; P=0.905$} & \multicolumn{2}{|c|}{$\chi^{2}=0.01 ; P=0.926$} \\
\hline \multicolumn{5}{|l|}{ Socioeconomic advantage/disadvantage } \\
\hline Most disadvantaged quartile & 676 & 21.3 & 148 & 52.0 \\
\hline Disadvantaged quartile & 858 & 21.1 & 203 & 52.2 \\
\hline Advantaged quartile & 822 & 17.0 & 187 & 42.3 \\
\hline \multirow[t]{2}{*}{ Most advantaged quartile } & 569 & 20.9 & 172 & 47.7 \\
\hline & \multicolumn{2}{|c|}{$\chi_{\text {trend }}^{2}=1.0 ; P=0.324$} & \multicolumn{2}{|c|}{$\chi_{\text {trend }}^{2}=1.9 ; P=0.171$} \\
\hline
\end{tabular}

over time, with almost half of the 2004 high-need group using genetic counseling; however, there are still half of those who might benefit from genetic counseling not attending the service.

The level of need for genetic counseling was the strongest predictor of utilization, with the high-need group 20 times more likely to use genetic counseling than the low-need group, and the moderate-need group three and a half times more likely. This finding is consistent with results from earlier studies in
Victoria $^{6,10}$ and other countries. ${ }^{5,7-9}$ Over time it is the increase in utilization by the high-need group, rather than the low-need or moderate-need groups, that is responsible for the overall increase in genetic counseling in Victoria. This finding is reassuring in terms of appropriate service provision.

After need, infant death (at $\geq 20$ weeks gestation or postnatally) was the next strongest predictor overall, with those experiencing stillbirth or postnatal death more than twice as likely to 
use genetic counseling than those whose infant was alive at hospital discharge, regardless of need or any other factor. This finding indicates that after the death of an infant, parents and/or their referring health professionals often perceive a benefit from follow-up support in the form of genetic counseling. Other studies have also found markedly increased levels of utilization

For each country/region of birth, utilization is shown as a \% of the total number of cases with that maternal country/region of birth and need (shown by the numbers above bars).

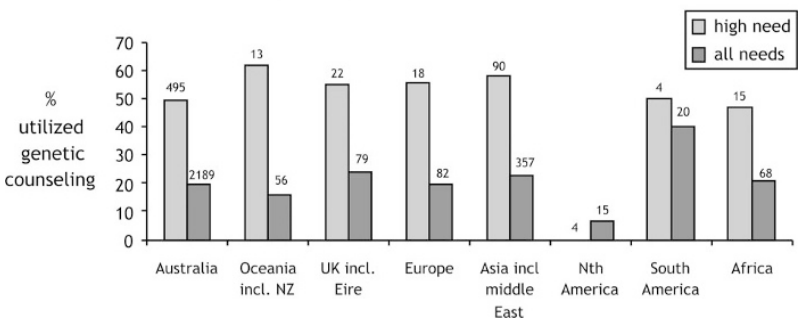

Fig. 2. Maternal country/region of birth and percent using genetic counseling, 2004 cohort (ascertained from Victorian Birth Defects Register). by families experiencing postnatal death ${ }^{5,7-9}$ and stillbirth ${ }^{4,5,7,8}$ compared with families of live born babies who survived. In the high-need group, only those experiencing postnatal death were more likely to use genetic counseling than other categories of infant survival status, but the effect of postnatal death not as pronounced as it was in the whole cohort.

In contrast, those having a termination of pregnancy $(<20$ weeks) were the least likely of the infant survival categories to use genetic counseling, particularly in the high-need group. This was different from the 1995 cohort where the termination of pregnancy $(<20$ weeks) group had the highest utilization at $53.2 \% .{ }^{10}$ The apparent under-utilization by the 2004 cohort may be due to genetic counseling provided outside of the state-wide service, with at least one genetic counselor now employed in the private sector for genetic counseling associated with prenatal diagnosis and screening. In addition, staff of the state-wide genetic counseling service anecdotally reported that obstetricians and fetal management multidisciplinary clinics based in maternity hospitals are increasingly providing genetic counseling, especially for the more commonly diagnosed conditions. This observation is consistent with our finding that the majority of the high-need, termination of pregnancy ( $<20$ weeks) group

Table 4 Logistic regression model assessing independent predictors of utilization of genetic counseling by 2004 birth defects cohort ascertained from Victorian Birth Defects Register

\begin{tabular}{|c|c|c|c|c|c|c|}
\hline \multirow[b]{2}{*}{ Variable } & \multicolumn{3}{|c|}{ Whole cohort } & \multicolumn{3}{|c|}{ High-need group } \\
\hline & Odds ratio & $95 \% \mathrm{CI}$ & $P$ & Odds ratio & $95 \% \mathrm{CI}$ & $P$ \\
\hline \multicolumn{7}{|l|}{ Need for counseling } \\
\hline Low & Ref & & $<0.001$ & & & \\
\hline Moderate & 3.5 & $2.5-4.9$ & & & & \\
\hline High & 20.7 & $14.4-29.8$ & & & & \\
\hline \multicolumn{7}{|l|}{ Maternal age } \\
\hline$<20$ & 1.7 & $0.8-3.5$ & 0.041 & 1.7 & $0.6-5.5$ & 0.016 \\
\hline $20-24$ & 1.4 & $0.8-2.3$ & & 0.8 & $0.4-1.8$ & \\
\hline $25-29$ & 1.4 & $0.9-2.2$ & & 0.8 & $0.5-1.5$ & \\
\hline $30-34$ & 1.9 & $1.3-2.8$ & & 1.9 & $1.1-3.1$ & \\
\hline $35-39$ & 1.4 & $0.9-2.1$ & & 1.2 & $0.8-2.0$ & \\
\hline $40+$ & Ref & & & Ref & & \\
\hline \multicolumn{7}{|l|}{ Type of birth hospital } \\
\hline Tertiary & Ref & & $<0.001$ & Ref & & $<0.001$ \\
\hline Metropolitan public & 0.5 & $0.4-0.6$ & & 0.4 & $0.3-0.6$ & \\
\hline Private & 0.4 & $0.3-0.5$ & & 0.3 & $0.2-0.4$ & \\
\hline Country & 0.4 & $0.3-0.6$ & & 0.3 & $0.2-0.4$ & \\
\hline \multicolumn{7}{|l|}{ Infant survival status at hospital discharge } \\
\hline Alive & Ref & & $<0.001$ & Ref & & $<0.001$ \\
\hline Termination of pregnancy ( $<20 \mathrm{wks}$ ) & 0.9 & $0.7-1.2$ & & 0.4 & $0.3-0.6$ & \\
\hline Stillbirth $^{a}$ & 2.5 & $1.6-3.9$ & & 0.7 & $0.4-1.3$ & \\
\hline Postnatal death ${ }^{b}$ & 2.9 & $1.9-4.6$ & & 1.6 & $0.8-3.0$ & \\
\hline
\end{tabular}


not using genetic counseling had a diagnosis of chromosomal trisomy or neural tube defect.

It is likely that this new direction in the provision of genetic counseling to women having a termination of pregnancy $(<20$ weeks) relates to an increase in the number of birth defects detected before 20 weeks gestation due to the availability of maternal serum screening since 1996/7 to pregnant women in Victoria. Between 1992 and 2002, there was a 5-fold increase in the proportion of prenatal diagnostic tests performed because of an "increased risk" screening result, with a significant increase in the number of major chromosomal abnormalities detected through prenatal diagnostic testing, from 3\% (115 cases) of all fetal chromosomal tests in 1992 to $5.5 \%$ (272 cases) in $2002 .{ }^{13}$ In 2004 , the number of pregnancies screened for birth defects in Victoria equated to $54 \%$ of live births. ${ }^{14}$ The increase in screening, and subsequent testing, during pregnancy is one facet of a generalized increase in genetic testing that has been associated with an increased need for genetic counseling. This has been observed elsewhere and said to be out of step with the supply of trained counselors, and for which solutions need to be found. ${ }^{15}$

Previous studies that have examined the association between maternal age and utilization of genetic counseling have variously found either that advanced maternal age is associated with increased utilization ${ }^{6,9}$ or with reduced utilization. ${ }^{8}$ After adjusting for all variables, our finding that women of advanced maternal age do not have an increased likelihood of using genetic counseling may be explained by the possibility that many of this group will have finished child-bearing, and consequently they, or their clinicians, may perceive that genetic counseling has limited relevance to them, although the intention to have a subsequent pregnancy is not necessarily associated with utilization of genetic counseling. ${ }^{16}$

The type of birth hospital was associated with utilization, with families at least twice as likely to use genetic counseling if the birth hospital was a tertiary level hospital compared with other hospital types. This may be related to the fact that all three tertiary level birth hospitals in Victoria have a genetic counseling service within the hospital, a situation likely to result in an increased awareness of genetic counseling by hospital staff and ease in making referrals. This theory is supported by additional analysis of utilization according to the location of the genetic clinic relative to the birth hospital (data not shown), which showed that utilization was higher for metropolitan residents when there was a genetic clinic located in the birth hospital. The location of the genetic clinic within the birth hospital may also be convenient for families using that birth hospital, but convenience of physical access is only part of the picture, as those giving birth in private hospitals located in the same building as a tertiary level hospital (and associated genetic clinic) had much lower levels of utilization. Interestingly, the distance of the nearest genetics clinic from the birth hospital was only associated with utilization for metropolitan residents, who were less likely to use genetic counseling with increasing distance. Perhaps, rural residents are more used to having to travel to access the services they need.

Those giving birth in private hospitals or country hospitals had the lowest utilization, which could be due to reduced awareness of the genetic counseling service or to these families being counseled privately. As most individuals/families are referred for genetic counseling by their primary care doctor or another specialist ${ }^{17}$ any lack of awareness of the availability or appropriateness of genetic counseling is likely to stem from the treating clinician. A survey of physicians about genetic services found that the main reason for nonreferral was a belief that it would not be of much benefit to the patient and concluded that many primary care physicians need more education about the genetic component of conditions to refer appropriately for genetics services. ${ }^{18}$

We examined a number of variables that may reflect access issues, as other studies have suggested that utilization may be adversely affected by location of clinics ${ }^{4,8}$ or racial origin. ${ }^{9}$ It was pleasing to find no evidence of such inequities. In particular, utilization of genetic counseling was not associated with area of residence or maternal country of birth; nor was it associated with socioeconomic advantage/disadvantage, although we acknowledge this measure is an estimate based on the post code of residence and not the individual families per se. None of these variables were included in the multivariate analysis because they were clearly not significant in the bivariate analysis.

Maternal country of birth was previously analyzed in Victoria for 1991 and 1993 cohorts and was not associated with utilization of genetic counseling at that time either. ${ }^{6}$ Area of residence was analyzed previously in Victoria (1995 cohort), at which time rural/regional residents used genetic counseling less than metropolitan residents, with utilization of 16.8 and $20.7 \%$ respectively. ${ }^{10}$ The increased utilization by rural/regional residents in the 2004 cohort to match that of their metropolitan counterparts may reflect the success of increasing the number of rural/regional towns having a genetics clinic from 5 in 1996/7 to 10 by 2004 , although additional clinics were not found to increase utilization in the Netherlands. ${ }^{7}$

Even though need was a strong predictor of utilization, it is important to note that just over half of the high-need families did not use the genetic counseling service. Although this may be appropriate if families made this choice themselves, or if they were provided with adequate genetic counseling by health practitioners outside of the specialized state-wide service, it is of concern if lack of awareness is the underlying factor. Previous studies have found that where there is an indication for genetic counseling, the key reasons people give for nonutilization are their own assessment about the usefulness of genetic counseling, or lack of awareness of the availability/appropriateness of genetic counseling. ${ }^{16,19}$ From the data available in this study, we cannot distinguish between families who fall into these two different categories.

The true proportion of all birth defects families using genetic counseling may be overestimated or underestimated by this study. A small overestimation is likely as the state-wide genetic service is one of the notifiers of birth defects to the Victorian Birth Defects Register; however, it was the sole source of notification in only 28 cases of the 2939 studied. On the other hand, a considerably greater underestimation of utilization is likely as data from one relatively new genetics clinic were not recorded on the genetic counseling database, a fact not known to the researchers at the time of doing the study. The genetics clinic concerned is located in a tertiary level hospital where 288 of the cases in the 2004 cohort were born; however, the utilization (elsewhere) by these families ascertained by the study was exceptionally low $(6.9 \%)$, probably because a number of them had genetic counseling at their birth hospital.

\section{CONCLUSIONS}

This record linkage study has demonstrated that during a 13-year period the utilization of genetic counseling by those who most need it is gradually increasing. The occurrence of a stillbirth or postnatal death across all categories of need seems to be strongly associated with utilization of genetic counseling. Reassuringly, there seems to be no inequity in access related to 
rural residence or social disadvantage, but further studies are needed to determine whether the $51.6 \%$ of high-need families not using genetic counseling are not doing so because of chance or choice.

\section{ACKNOWLEDGMENTS}

This work was supported by Department of Human Services, Victoria and by National Health and Medical Research Council, fellowship number 436904 (to J.H.).

The authors thank the Victorian Perinatal Data Collection Unit, Department of Human Services for providing data for record linkage from the Victorian Birth Defects Register; and Genetic Health Services Victoria for providing data for record linkage from its genetic counseling database.

\section{REFERENCES}

1. Brent RL. Environmental causes of human congenital malformations: the pediatrician's role in dealing with these complex clinical problems caused by a multiplicity of environmental and genetic factors. Pediatrics 2004;113 (4 suppl):957-968.

2. Hassed SJ. Genetic counseling. J Okla State Med Assoc 2003;96:495-497.

3. Fryer AE. Clinical genetic services: activity, outcome, effectiveness and quality. Summary of a Report of the Clinical Genetics Committee of the Royal College of Physicians. J R Coll Physicians Lond 1997;31:624- 627.

4. Julian C, Tordo MC, Macquart-Moulin G, et al. Factors influencing genetic counseling attendance rate: a geographically based study. Soc Biol 1989;36: $240-247$.

5. Cornel MC, van Essen AJ, ten Kate LP. Comparison of couples referred and not referred for genetic counseling in a genetic clinic after the birth of a child with a congenital anomaly: a study in a population in the northeastern Netherlands. Am J Med Genet 1992;42:387-392.
6. Halliday J, Griffin O, Bankier A, et al. Use of record linkage between a statewide genetics service and a birth defects/congenital malformations register to determine use of genetic counselling services. Am J Med Genet 1997;72:3-10.

7. Sikkens EH, de Walle HEK, Reefhuis J, et al. Referral for genetic counseling after the birth of a child with a congenital anomaly in the northern Netherlands. Am J Med Genet 2002;112:133-137.

8. Mammi I, Basile RT, Bellato S, et al. Utilization of genetic counseling by parents of a child or fetus with congenital malformation in north-east Italy. Am J Med Genet 2003;121:214-218.

9. Forrester MB, Merz RD. Genetic counseling utilization by families with offspring affected by birth defects, Hawaii, 1986-2003. Am J Med Genet A 2007;143A:1045-1052

10. Collins V. the changing face of genetics services in Victoria [dissertation]. Melbourne: The University of Melbourne, 2003.

11. Riley M, Phyland S, Halliday J. Validation study of the Victorian birth defects register. J Paediatr Child Health 2004;40:544-548.

12. Trewin D. Australian Bureau of Statistics, Socio-Economic Indexes for Areas: Australian Bureau of Statistics, 2001. Available at: www.abs.gov.au.

13. Muggli EE, Halliday JL. Prenatal diagnostic testing and Down syndrome in Victoria 1992-2002. Aust N Z J Public Health 2004;28:465-470.

14. O'Leary P, Breheny N, Reid G, et al. Regional variations in prenatal screening across Australia: stepping towards a national policy framework. Aust N Z J Obstet Gynaecol 2006;46:427-432.

15. Frontali M, Jacopini AG. Genetic counselling: evolution or involution? Commun Genet 2000;3:175-178.

16. Hobus I, Frets PG, Duivenvoorden HJ, et al. Factors influencing whether or not couples seek genetic counselling: an explorative study in a paediatric surgical unit. Clin Genet 1995;47:47-52.

17. Donnai D. Genetic services. Clin Genet 2002;61:1-6.

18. Hayflick SJ, Eiff MP, Carpenter L, Steinberger J. Primary care physicians' utilization and perceptions of genetics services. Genet Med 1998;1:13-21.

19. Collins V, Halliday J, Williamson R. What predicts the use of genetic counseling services after the birth of a child with Down syndrome? J Genet Counsel 2003;12:43-60. 